Pacific Journal of Mathematics

ON THE ESSENTIAL SPECTRUM OF SCHROEDINGER
OPERATORS WITH SINGULAR POTENTIALS 


\title{
ON THE ESSENTIAL SPECTRUM OF SCHROEDINGER OPERATORS WITH SINGULAR POTENTIALS
}

\author{
JAMES S. HowLAND
}

\begin{abstract}
In this paper, we show that under certain conditions the self-adjoint Schroediner operator $-\Delta_{n}+V(x)$ on $L_{2}\left(R_{n}\right), n \geqq 1$, has essential spectrum $[0, \infty)$. The theorems improve previous results by permitting $V(x)$ to be more singular locally. The proof employs a factorization $V(x)=A(x) B(x)$ of the potential.
\end{abstract}

The essential spectrum of a self-adjoint operator is defined to consist of all points of the spectrum which are not isolated eigenvalues of finite multiplicity. Let $V(x)$ be a real-valued function on the $n$ dimensional Euclidian space $R_{n}$, and $\Delta_{n}$ the $n$-dimensional Laplacian. In a recent paper [6], Rejto gives conditions on $V$ such that the operator $T=-\Delta_{n}+V(x)$ is self-adjoint with domain $\mathscr{D}(T)=\mathscr{D}\left(\Delta_{n}\right)$ and has essential spectrum $[0, \infty)$. His method consits essentially in proving compactness of the operator $V R_{0}(z)$, where $R_{0}(z)=\left(-\Delta_{n}-z\right)^{-1}$. The condition $\mathscr{D}(V) \supseteq \mathscr{D}\left(\Delta_{n}\right)$, which accounts for the equality of the domains of $T$ and $A_{n}$, is essential to this method, and corresponds very roughly to local square-integrability of $V(x)$.

Recent papers of Kato [3] and Kuroda [5] on the continuous spectrum and of Konno and Kuroda [4] on the discrete spectrum employ a method according to which one factors the potential into $V(x)=A(x) B(x)$ and considers the operator $A R_{0}(z) B$. In this theory, the operator $T$ is defined by first defining its resolvent, and there is no guarantee that the domains of $T$ and $\Delta_{n}$ are equal. This is rather an advantage, since it removes the requirement of local square-integrability. For example, Kato [3, §6] shows that if, for $n=3$, the norm of $V$ in $L_{3 / 2}\left(R_{3}\right)$ is sufficiently small, then $T$ is unitarily equivalent to $-\Delta_{3}$.

In the present paper, we shall apply the factorization technique to the problem of invariance of the essential spectrum, extending Rejto's results to include potentials which are locally more singular. In particular, we remove certain seemingly artificial restrictions of [6] in the case of low $(n \leqq 3)$ dimensions. For $n=3$, our results will apply to $V(x)=|x|^{-a}$ for any $a<2$.

For $n \geqq 3$, the definition and semi-boundedness of $T$ are treated in $\S 1$, and the essential spectrum in $\S 2 . \quad \S 3$ is devoted to the proofs of the essential estimates for $n \geqq 3$. The special cases $n=1,2$ are discussed in $\$ 4$.

For references to other work on essential spectra, we refer to the extensive bibliography of [6]. 
1. Definition and semi-boundedness. In order to define precisely the Schroedinger operator $-\Delta_{n}+V(x)$, we shall use the following theorem of Kato [3], as formulated by Konno and Kuroda [4]. $\mathscr{X}(T)$, $\sigma(T)$ and $\rho(T)$ denote respectively the domain, spectrum and resolvent set of the operator $T$.

Lemma 1.1 (Kato). Let $T_{0}$ be a self-adjoint operator on a separable Hilbert space $\mathscr{X}$, and $R_{0}(z)=\left(T_{0}-z\right)^{-1}$ its resolvent. Let $A$ and $B$ be closed, densely defined operators on $\mathscr{C}$ such that

$$
\mathscr{D}\left(T_{0}\right) \subseteq \mathscr{D}(A) \cap \mathscr{D}(B)
$$

and

$$
(A x, B y)=(B x, A y)
$$

for all $x, y$ in $\mathscr{D}(A) \cap \mathscr{D}(B)$. Suppose that for some $z$ in $\rho\left(T_{0}\right)$, the operator $A R_{0}(z) B$ has a bounded extension to $\mathscr{Z}$ such that $\max \left\{\left\|A R_{0}(z) B\right\|,\left\|A R_{0}(\bar{z}) B\right\|\right\}<1$.

Then $A R_{0}(z) B$ has a bounded extension for every $z$ in $\rho\left(T_{0}\right)$ and there exists a self-adjoint extension $T$ of $T_{0}+B^{*} A$ such that if $z \in \rho\left(T_{0}\right)$ and $I+A R_{0}(z) B$ has a bounded inverse, then $z \in \rho(T)$ and

$$
R(z)=R_{0}(z)-\left[B R_{0}(\bar{z})\right]^{*}\left(I+A R_{0}(z) B\right)^{-1} A R_{0}(z) .
$$

where $R(z)=(T-z)^{-1}$.

The operator $T_{0}=-\Delta_{n}$ is self-adjoint on $\mathscr{C}=L_{2}\left(R_{n}\right)$ and has purely continuous spectrum $\sigma\left(T_{0}\right)=[0, \infty)$. If $n \geqq 3$, then for $z$ in $\rho\left(T_{0}\right)$ the resolvent $R_{0}(z)=-\left(\Delta_{n}+z\right)^{-1}$ is convolution by a function

$$
g_{n}(x, k)=|x|^{2-n} F_{n}(k|x|)
$$

where $k^{2}=z, \operatorname{Im} k \geqq 0 . \quad F_{n}(z)$ can be expressed in terms of a Hankel function $[7$, p. 79 , formula (13.7.2)] and satisfies the following inequality for $\operatorname{Im} z \geqq 0$

$$
\left|F_{n}(z)\right| \leqq C_{n}\left(1+|z|^{(n-3) / 2}\right) \exp (-\operatorname{Im} z)
$$

where $C_{n}$ depends only on $n$. In particular, if $k=2 i \eta, \eta>0$, then

$$
\left|F_{n}(k|x|)\right| \leqq C_{n}^{\prime} e^{-\eta|x|}
$$

where $C_{n}^{\prime}=C_{n} \max \left\{\left(1+t^{(n-3) / 2}\right) e^{-t / 2}: 0 \leqq t<\infty\right\}$.

Let $B(x, \delta)$ be the closed ball with center $x$ and radius $\delta$. For any measurable function $g$ on $R_{n}$, we define for $1 \leqq p<\infty$ and $0<\delta \leqq 1$

$$
I_{p, \nu}(g, \delta)=\sup _{x \in R_{n}}\left(\int_{B(x, \delta)}|x-y|^{-\nu}|g(y)|^{p} d y\right)^{1 / p}
$$


and $I_{p, \nu}(g)=I_{p, \nu}(g, 1)$, a notation that differs from that of [6] in the sign of $\nu$. Observe that $I_{p, \nu}(g, \delta)$ is increasing in $\nu$ and $\delta$ and that if $p<q$ and $p q a=(n-\nu) p-(n-\nu-\varepsilon) q>0$, then by Holder's inequality

$$
I_{p, \nu+\varepsilon}(g, \delta) \leqq C \delta^{a} I_{q, \nu}(g, \delta)
$$

where $C$ depends on $n, p$ and $q$.

THEOREM 1. Let $V(x)$ be a real-valued, measurable function on $R_{n}, n \geqq 3$. Assume that there exists $a p, 1<p<\infty$ such that either

(a) $I_{p / 2, \nu}(V)<+\infty$ for some $\nu>n-p$

$a r$

(b) $I_{p / 2, \nu}(V)<+\infty$ for $\nu=n-p$ and

$$
\lim _{|x| \rightarrow \infty} \int_{B(x, \hat{o})}|x-y|^{p-n}|V(y)|^{p / 2} d y=0 .
$$

Then there exists a self-adjoint extension $T$ of $-\Delta_{n}+V(x)$ which is bounded below.

Proof. Let $A$ and $B$ be the multiplication operators defined by the functions $A(x)=|V(x)|^{1 / 2}$ and $B(x)=|V(x)|^{1 / 2} \operatorname{sgn} V(x)$. If $k=2 i \eta$, $\eta \geqq \eta_{0}>0$, then by (3.3) the operator $B R_{0}\left(k^{2}\right)$ is bounded, which implies that $\mathscr{D}(B)=\mathscr{D}(A) \supseteqq \mathscr{D}\left(T_{0}\right)$. Furthermore, by (3.2),

$$
\left\|A R_{0}\left(k^{2}\right) B\right\| \leqq C\left[I_{p / 2, \nu}(V, \delta) \delta^{a}+I_{p / 2, \nu}(V) \delta^{2-n} \eta^{-b}\right]
$$

where $a p=p+\nu-n, b p=n(p-2)$ and $C$ depends on $n, \nu, p$ and $\eta_{0}$. Note that $b>0$, while $a>0$ in case (a) and $a=0$ in case (b). Choose a fixed $\delta$ sufficiently small that the first term on the right is less than unity. (In case (b), where $a=0, I_{p / 2, \nu}(V, \delta) \rightarrow 0$ as $\delta \rightarrow 0+$. This can be proved by using (1.3) to restrict consideration to a bounded set, where absolute continuity yields the result.) Then for all sufficiently large $\eta$, we have

$$
\left\|A R_{0}\left(k^{2}\right) B\right\|<1
$$

so that the theorem follows from Lemma 1.1.

REMARKS (1) In the special case $\nu=0$, we obtain $p>n$ in condition (a), so that if $V$ is a function of $r=|x|$ only, the result applies to singularities at the origin of order $r^{-\alpha}$ for $\alpha<2$. If $u(r)$ is a spherically symmetric $L_{2}\left(R_{n}\right)$-eigenfunction of $T$ with eigenvalue $\lambda$, 
then $v=r^{(n-1) / 2} u$ is an $L_{2}\left(R_{1}\right)$-solution of the ordinary differential equation

$$
-v^{\prime \prime}+\left[V(r)+c_{n} / r^{2}\right] v=\lambda v
$$

where $c_{n}=(n-1)(n-3) / 4$. However, [2, Vol. 2, pp. 1462-9, esp. Th. XIII. 7.40] if

$$
\lim _{r \rightarrow 0} \sup _{r} r^{2} V(r)<-c_{n}-1 / 4
$$

it follows that (1.4) has a point spectrum which is unbounded below. Thus if $V(r)$ has a singularity at the origin of order $r^{-\alpha}, \alpha \geqq 2$, there may be no lower semibounded extension of $-\Delta_{n}+V$. Theorem 1 therefore seems near the "best" with regard to the local behavior of $V$.

(2) Note that if $\nu<0$, then

$$
\int_{B(x, \tilde{o} / 3)}|g(y)| d y \leqq(6 / \delta)^{-\nu} \int_{B\left(x_{0}, \tilde{o}\right)}\left|x_{0}-y\right|^{-\nu}|g(y)| d y
$$

whenever $\left|x-x_{0}\right|=\delta / 2$. Hence, negative values of $\nu$ add noting to Theorem 1 , and one might as well assume that $\nu \geqq 0$. A similar remark holds for Theorem 2 of the next section.

2. The essential spectrum. The essential spectrum $\sigma_{e}(T)$ of a self-adjoint operator $T$ is defined to consist of those points of $\sigma(T)$ which are not isolated eigenvalues of finite multiplicity. Two selfadjoint operators $T$ and $T_{0}$ are resolvent congruent if and only if $R_{0}(z)-R(z)$ is compact for some $z$ in $\rho(T) \cap \rho\left(T_{0}\right)$. It is well-known that if $T$ and $T_{0}$ are resolvent congruent, then $\sigma_{e}(T)=\sigma_{e}\left(T_{0}\right)$. We refer to [6] for references.

Lemma 2.1. Let $T$ and $T_{0}$ be as in Lemma 1.1 and assume that $A R_{0}(z) B$ and $\left[B R_{0}(\bar{z})\right]^{*} A R_{0}(z)$ are compact for some $z$ in $\rho(T) \cap \rho\left(T_{0}\right)$. Then $T$ and $T_{0}$ are resolvent congruent, and hence $\sigma_{e}(T)=\sigma_{e}\left(T_{0}\right)$.

The proof is immediate from (1.1) and the identity

$$
(I+K)^{-1}=I-K(I+K)^{-1} \text { with } K=A R_{0}(z) B .
$$

THEOREM 2. Let $V(x)$ be a real-valued measurable function on $R_{n}, n \geqq 3$, for which there exist $p$ and $\nu, 2<p<\infty$ and $\nu>n-p$, such that

$$
\int_{B(x, 1)}|x-y|^{-2}|V(y)|^{p / 2} d y
$$

is bounded on $R_{n}$ and vanishes as $|x|$ tends to infinity. If $T$ is the 
extension of $-\Delta_{n}+V$ defined in Theorem 1 , then $\sigma_{e}(T)=[0, \infty)$.

Proof. Define $A$ and $B$ as in the proof of Theorem 1. We shall first prove that $A R_{0}\left(k^{2}\right) B$ is compact for $k=2 i \eta, \eta>0$. Let $S$ denote the image under $A R_{0}\left(k^{2}\right) B$ of the unit ball of $L_{2}\left(R_{n}\right)$. We shall show that $S$ is pre-compact. For every $f=A R_{0}\left(k^{2}\right) B u,\|u\| \leqq 1$ in $S$, we have, by Lemma 3.2 ,

$$
\int_{|x| \geq N}|f(x)|^{2} d x \leqq C I_{p, \nu}\left(\chi_{B(0, N)} A\right)
$$

where $C$ is independent of $f$ in $S$, and $\chi$ denotes the characteristic function. Since $I_{p, \nu}(A, x)$ vanishes at infinity, the right side of (2.2) vanishes as $N$ tends to infinity. It therefore suffices by Rellich's Theorem [2, Vol. 1, Th. IV. 8.21] to prove that $S$ is equicontinuous in $L_{2}$-norm.

For an arbitrary $f$, let $f_{h}(x)=f(x-h)$ and write $R_{0}\left(k^{2}\right) u=g * u$, where * denotes convolution. Then for every $f=A R_{0} B u$ in $S$, we have

$$
\left\|f_{h}-f\right\| \leqq\left\|A\left(g_{h}-g\right)(B u) *\right\|+\|\left(A_{h}-A\right) g_{h} *(B u \| \text {. }
$$

Let $\varphi(x, h)=e^{-\eta|x+h|}-e^{-\eta|x|}$. Then for each fixed $x, \varphi(x, h)$ vanishes as $h$ tends to zero and does not exceed $1+e^{\eta}$ in absolute value for $|h| \leqq 1$. In order to estimate $\left\|A\left(g_{h}-g\right) *(B u)\right\|$, one repeats the arguments of Lemma 3.2 (with, say, $\delta=1$ ), carrying along a factor $|\varphi(x-y, h)|$. We obtain as a result that for $\|u\| \leqq 1$ and $|h| \leqq 1$, \| $A\left(g_{h}-g\right) *(B u) \|$ does not exceed

$$
C\left(\int_{B(x, 1)}|x|^{-\beta}|\varphi(x, h)|^{a} d x\right)^{1 / a}+C \int_{R_{n}} e^{-\eta|x|}|\varphi(x, h)| d x
$$

where $C$ is independent of $u$ and $h, \beta=(n-2-\nu) \alpha+\nu$ and $a=p /(p-2)$. As $h$ tends to zero, these terms vanish.

Choose $\mu$ and $q$ such that $2<q<p$ and $n-q<\mu<\nu$. Then since $I_{p, \nu}(A, x)$ is increasing in both subscripts, $I_{q, \mu}(A, x)$ is also bounded and vanishes at infinity. Moreover, we claim that $I_{q, \mu}\left(A_{h}-A\right)$ vanishes as $h$ tends to zero. For since $I_{q, \mu}(A, x)$ vanishes at infinity, we may assume that $A$ has compact support $K$. In this case $A$ is in $L_{p}\left(R_{n}\right)$, since

$$
\|A\|_{p} \leqq C(K) I_{p, 0}(A) \leqq C(K) I_{p, \nu}(A)
$$

where $C(K)$ is the number of balls of unit radius necessary to cover $K+B(0,1)$. Hence by $(3.4)$,

$$
I_{q, \mu}\left(A_{h}-A\right) \leqq C I_{p, 0}\left(A_{h}-A\right) \leqq C\left\|A_{h}-A\right\|_{p}
$$


which vanishes as $h$ tends zero. We now observe that by Lemma 3.2,

$$
\left\|\left(A_{h}-A\right) g *(B u)\right\| \leqq C I_{q, \mu}\left(A_{h}-A\right)
$$

where $C$ is independent of $f$ in $S$. Hence $S$ is equicontinuous in $L_{2}$ norm and $A R_{0}\left(k^{2}\right) B$ is compact.

If we now remark that $A R_{0}\left(k^{2}\right)$ is compact by the results of [6, $\S 2]$, then Theorem 2 follows from Lemma 2.1. Alternatively, Lemma 4.26 and the above procedure can be used to obtain a proof of the compactness of $R_{0}\left(k^{2}\right) A$ and hence of its adjoint $A R_{0}\left(k^{2}\right)$. The proof is simpler, since the second part of the argument-that involving $\left(A_{h}-A\right)$-does not occur. This method also yields a proof of Theorem 2.1 of [6] which avoids approximation by smoothed resolvent kernels.

REMARK In Theorem 2, it suffices to assume that for some $p, \nu$, $2<p<\infty$ and $\nu>n-p$ the function (2.1) is bounded on $R_{n}$ and that for some $\mu>0$

$$
\lim _{|x| \rightarrow \infty} \int_{B(x)}|V(q)|^{\mu} d y=0 .
$$

This assumption is similar to that of [6].

For the proof, assume without loss of generality, that $0<2 \mu<p$, and let $0 \leqq s, t \leqq 1, s+t=1$. Writing $p^{\prime} / 2=\left(p^{\prime} / 2-\mu s\right)+\mu s$, we obtain by Holder's inequality

$$
\int_{B(x)}|x-y|^{-\nu^{\prime}}|V(y)|^{p^{\prime} / 2} d y \leqq\left(\int_{B(x)}|V(y)|^{\mu} d y\right)^{s}\left(I_{p / 2, \nu}(V)\right)^{t}
$$

where $p^{\prime}=t p+\mu s<p$ and $\nu^{\prime}=t \nu<\nu$. By choosing $s$ sufficiently small, the inequality $\nu^{\prime}>n-p^{\prime}$ can be made to hold, so that the hypotheses of Theorem 2 are satisfied for $p^{\prime}$ and $\nu^{\prime}$.

3. The basic estimates for $n \geqq 3$. The quantities $I_{p, \nu}(g, \delta)$ and $I_{p, \nu}(g)$ have been defined in $\S 1$.

LEMmA 3.1. If $0<\eta_{0} \leqq \eta$ and $n \geqq 1$, then

$$
\sup _{x \in R_{n}} \int_{R_{n}} e^{-\eta|x-y|}|f(y)| d y \leqq C\left(\nu, \eta_{0}\right) I_{1, \nu}(f) \text {. }
$$

Proof. For a fixed $x$, we have

$$
\int_{R_{n}} e^{-\eta|x-y|}|f(y)| d y \leqq \sum_{N=0}^{\infty} e^{-\eta N}(N+1)^{\nu} \int_{A(N, x)}|x-y|^{-\nu}|f(y)| d y
$$

where $A(x, N)$ is the " annulus" $B(x, N+1) \sim B(x, N)$. If $B(\xi, 1)$ is a ball of unit radius with center $\xi$ in $A(N, x)$ then for $N \geqq 1$ 


$$
\int_{B(\xi, 1)}|x-y|^{-\nu}|f(y)| d y \leqq \int_{B(\xi, 1)}|\xi-y|^{-\nu}|f(y)| d y \leqq I_{1, \nu}(f) .
$$

Since $A(x, N)$ can be covered by $O\left(N^{n-1}\right)$ such balls, the right side of (3.1) does not exceed

$$
C I_{1, \nu}(f) \sum_{N=0}^{\infty} e^{-\eta N}(N+1)^{\nu+n-1}
$$

where the sum is bounded uniformly for $\eta \geqq \eta>0$.

LEMmA 3.2. Let $A(x)$ and $B(x)$ be measurable on $R_{n}$, $n \geqq 3$, $k=2 i \eta, \eta \geqq \eta_{0}>0$ and $0<\delta<1$.

(a) If $2<p<\infty$ and $p+\nu \geqq n$, then

$$
\left\|A R_{0}\left(k^{2}\right) B\right\| \leqq C\left[I_{p, \nu}(A, \delta) I_{p, \nu}(B, \delta) \delta^{a}+I_{p, \nu}(A) I_{p, \nu}(B) \delta^{2-n} \eta^{-b}\right]
$$

where $a p=2(p+\nu-n), p b=n(p-2)$ and $C$ depends on $n, \nu, p$ and $\eta_{0}$.

(b) If $2 \leqq p<\infty$ and $\nu+2 p \geqq n$, then

$$
\left\|B R_{0}\left(k^{2}\right)\right\|=\left\|R_{0}\left(k^{2}\right) B\right\| \leqq C\left[I_{\nu, p}(B, \delta) \delta^{r}+\delta^{2-n} I_{\nu, p}(B) \eta^{-n}\right]
$$

where $r p=2 p+\nu-n$, and $C$ depends on $n, \nu, p$ and $\eta_{0}$.

Before proceeding with the proof, we remark for reference the elementary inequality

$$
\|f * g\|_{p} \leqq\|f\|_{1}\|g\|_{p}
$$

where $*$ denotes convolution and $\|\cdot\|_{p}$ is the norm of $L_{p}\left(R_{n}\right)$.

Proof. For part (a), let $u \in L_{2}\left(R_{n}\right)$. Then by (1.2)

$$
\begin{aligned}
\left|R_{0}\left(k^{2}\right) B u(x)\right| & \leqq C \int_{B(x, \delta)}|x-y|^{2-n}|B(y) u(y)| d y \\
& +\delta^{2-n} \int_{R_{n}} e^{-\eta|x-y|}|B(y) u(y)| d y
\end{aligned}
$$

where $C$ depends only $n$ and $\eta_{0}$.

Let $2 \leqq p<\infty$ and $p^{-1}+q^{-1}=1$. Using Holder's inequality with respect to the measure $|x-y|^{-\nu} d y$, we find that the first term of (3.5) does not exceed

$$
I_{p, \nu}(B, \delta)\left(\int_{B(x, \delta)}|x-y|^{-\alpha}|u(y)|^{q} d y\right)^{1 / q}
$$

where $\alpha=(n-2-\nu) q+\nu$. Multiply (3.6) by $A(x)$. The $L_{2}$-norm of the resulting function is, except for a factor $I_{p, \nu}(B, \delta)$, the $L_{2 / q}$-norm of 


$$
f(x)=|A(x)|^{q} \int_{B(x, \tilde{s})}|x-y|^{-\alpha}|u(y)|^{q} d y .
$$

Let $v \in L_{2 / q}^{*}=L_{2 /(2-q)}$ and form the inner product $(v, f)$. Interchanging the order of integration and applying Holder's inequality with respect to the measure $|x-y|^{-\nu} d x$, we obtain

$$
|(v, f)| \leqq\left[I_{p, \nu}(A, \delta)\right]^{q}\left(|u|^{q}, g^{1 / \sigma}\right)
$$

where

$$
\begin{aligned}
g(x) & =\int_{B(x, \partial)}|x-y|^{-\beta}|v(x)|^{\sigma} d x \\
(1 / \sigma)+(q / p) & =1 \text { and } \beta=(n-2-\nu) \sigma q+\nu .
\end{aligned}
$$

But

$$
\begin{aligned}
\left(|u|^{q}, g^{1 / \sigma}\right) & \leqq\left\|u^{q}\right\|_{2 / q}\left\|g^{1 / \sigma}\right\|_{2 /(2-q)} \\
& =\|u\|_{2}^{q}\|g\|_{2}^{1 / \sigma}
\end{aligned}
$$

while $\|g\|_{2} \leqq C \delta^{n-, \beta}\left\|v^{\sigma}\right\|_{2}=C^{n-\beta}\|v\|_{2 /(2-q)}^{\sigma}$, provided that $\beta \leqq n$. (If $n>\beta$, This follows from (3.4), while if $n=\beta$, one uses Sobolev's inequality $[1$, p. 220 , part (c) $]$.) Combining all these estimates yields the first term on the right side of (3.2), provided we note that $\beta \leqq n$ if and only if $p+\nu \geqq n$ and that $a=(n-\beta) / \sigma q$.

If we apply Holder's inequality with respect to the measure $e^{-\eta|x-y|} d y$, we find that the integral in the second term of (3.5) does not exceed

$$
\left(\int_{R_{n}} e^{-\eta|x-y|}|B(y)|^{p} d y\right)^{1 / p}\left(\int_{R_{n}} e^{-\eta|x-y|}|u(y)|^{q} d y\right)^{1 / q} .
$$

By Lemma 3.1, the first factor does not exceed $C\left(\eta_{0}, \nu\right) I_{p},{ }_{\nu}(B)$. If the second factor is multiplied by $A(x)$, we find as above that it suffices to estimate

$$
\int_{R_{n}} v(x)|A(x)|^{q} \int_{R_{n}} e^{-\eta|x-y|}|u(y)| q d y d x
$$

where $v \in L_{2 \mid q}^{*}$. Interchange the order of integration and use Holder's inequality with respect to the measure $e^{-y|x-y|} d x$. The resulting expression is easily estimated using (3.4), and we find that the $L_{2}$-norm of the product of $A(x)$ with the second factor of (3.7) does not exceed $C I_{p, \nu}(A) \eta^{-b}$, where we note that $b=n / \sigma q$. This yields the second term of (3.2).

For part (b), return to (3.5). In the first term, observe that since $|u|^{q}$ is in $L_{2 / q}$, Holder's inequality implies that the $L_{2}$-norm of the second factor of (3.6) does not exceed 


$$
C^{(n-\alpha) / q}\|u\|_{2}
$$

for $\alpha<n$. For $\alpha=n$, the same is true by Sobolev's inequality [1, p. 220, part (c)]. In the second term, estimate the second factor of (3.7) by (3.4) and the first factor by Lemma 3.1. Combining these results yields (3.3), if we observe that $(n-\alpha) / q=r$, so that $\alpha \leqq n$ if and only if $\nu+2 p \geqq n$.

4. The cases $n=1$ and $n=2$. The cases of lowest dimension must be considered separately because of the different form of $g_{n}(x, k)$. For $n=2$, we have

$$
g_{2}(x, k)=(i / 4) H_{0}^{(1)}(k|x|)
$$

where $\left|H_{0}^{(1)}(i t)\right|$ does not exceed $t^{-1} e^{-t}$ for $t>0$, nor $\log |t|$ for $0<t \leqq 1$. Let $0<\delta \leqq 1 \leqq \eta$, and use the first estimate for $g_{2}(x, i \eta)$ for $\eta|x| \geqq \delta$ and the second for $\eta|x| \leqq \delta$. One then obtains estimates like those of $\S 3$, except that the use of Sobolev's Theorem in Lemma 3.2 is not justified, due to the presence of a logarithmic factor. This results in the failure of the proof of Theorem $1(\mathrm{~b})$ for $n=2$. Theorems 1(a) and 2 hold for $n=2$.

If $n=1$, we have

$$
g_{1}(x, k)=k^{-1} e^{i k|x|} .
$$

Since there is no singularity at $x=0$, only the second of the terms estimated in the proof of Lemma 3.2 appears, and the estimates are greatly simplified. As a result, Theorems 1 and 2 hold for $n=1$, without the hypothesis $p+\nu>1$; $\nu$ may be completely arbitrary. The case $n=1$ has been studied extensively by the special techniques of ordinary differential equations; see [2, Chapter XIII.].

\section{BIBLIOGRAPHY}

1. L. Bers, F. John, and M. Schecter, Partial differential equations, Lectures in Applied Mathematics, Vol. III, Interscience, 1964.

2. N. Dunford, and J. Schwartz, Linear Operators, Vol. 1, 1958; Vol. 2, 1963, Interscience.

3. T. Kato, Wave operators and similarity for some non-self-adjoint operators, Math. Ann. 162 (1966), 258-279.

4. R. Konno, and S. T. Kuroda, On the finiteness of perturbed eigenvalues, J. Fac. Sci., U. Tokyo, Sec. I, Vol. XIII, part 1, 1966.

5. S. T. Kuroda, An abstract stationary approach to perturbation of continuous spectra and scattering theory, J. Analyse Math. 20 (1967), 57-117.

6. P. A. Rejto, On the essential spectrum of the hydrogen energy and related operators, Pacific J. Math. 19 (1966), 109-140.

7. E. C. Titchmarsh, Eigenfunction Expansions Associated with Second Order Differential Operators, Vol. II, Oxford, 1950. 
Received August 17, 1967. This work was partly supported by NSF Institutional Grant GU-1978.

UNIVERSITY OF VIRGINIA

Charlottesville, Virginia 


\title{
PACIFIC JOURNAL OF MATHEMATICS
}

\author{
EDITORS
}

\section{H. ROYDEN}

Stanford University

Stanford, California

\author{
J. P. JANS \\ University of Washington \\ Seattle, Washington 98105
}

\section{J. DugundJI}

Department of Mathematics

University of Southern California

Los Angeles, California 90007

\section{RICHARD ARENS}

University of California

Los Angeles, California 90024

\section{ASSOCIATE EDITORS}
E. F. BECKENBACH
B. H. NEUMANN
F. WOLF
K. YOSIDA

\section{SUPPORTING INSTITUTIONS}

\author{
UNIVERSITY OF BRITISH COLUMBIA \\ CALIFORNIA INSTITUTE OF TECHNOLOGY \\ UNIVERSITY OF CALIFORNIA \\ MONTANA STATE UNIVERSITY \\ UNIVERSITY OF NEVADA \\ NEW MEXICO STATE UNIVERSITY \\ OREGON STATE UNIVERSITY \\ UNIVERSITY OF OREGON \\ OSAKA UNIVERSITY \\ UNIVERSITY OF SOUTHERN CALIFORNIA
}

\author{
STANFORD UNIVERSITY \\ UNIVERSITY OF TOKYO \\ UNIVERSITY OF UTAH \\ WASHINGTON STATE UNIVERSITY \\ UNIVERSITY OF WASHINGTON \\ AMERICAN MATHEMATICAL SOCIETY \\ CHEVRON RESEARCH CORPORATION \\ TRW SYSTEMS \\ NAVAL WEAPONS CENTER
}

Mathematical papers intended for publication in the Pacific Journal of Mathematics should be in typed form or offset-reproduced, double spaced with large margins. Underline Greek letters in red, German in green, and script in blue. The first paragraph or two must be capable of being used separately as a synopsis of the entire paper. It should not contain references to the bibliography. Manuscripts, in duplicate if possible, may be sent to any one of the four editors. All other communications to the editors should be addressed to the managing editor, Richard Arens, University of California, Los Angeles, California 90024.

Each author of each article receives 50 reprints free of charge; additional copies may be obtained at cost in multiples of 50 .

The Pacific Journal of Mathematics is published monthly. Effective with Volume 16 the price per volume (3 numbers) is $\$ 8.00$; single issues, $\$ 3.00$. Special price for current issues to individual faculty members of supporting institutions and to individual members of the American Mathematical Society: $\$ 4.00$ per volume; single issues $\$ 1.50$. Back numbers are available.

Subscriptions, orders for back numbers, and changes of address should be sent to Pacific Journal of Mathematics, 103 Highland Boulevard, Berkeley 8, California.

Printed at Kokusai Bunken Insatsusha (International Academic Printing Co., Ltd.), 7-17, Fujimi 2-chome, Chiyoda-ku, Tokyo, Japan.

PUBLISHED BY PACIFIC JOURNAL OF MATHEMATICS, A NON-PROFIT CORPORATION

The Supporting Institutions listed above contribute to the cost of publication of this Journal, but they are not owners of publishers and have no responsibility for its content or policies. 


\section{Pacific Journal of Mathematics}

\section{Vol. 25, No. $3 \quad$ November, 1968}

Philip Marshall Anselone and Theodore Windle Palmer, Collectively

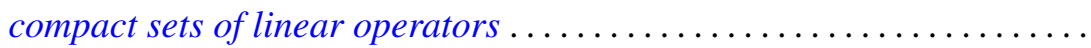

Philip Marshall Anselone and Theodore Windle Palmer, Spectral analysis of collectively compact, strongly convergent operator sequences.........

Edward A. Bender, Characteristic polynomials of symmetric matrices...... 433

Robert Morgan Brooks, The structure space of a commutative locally convex

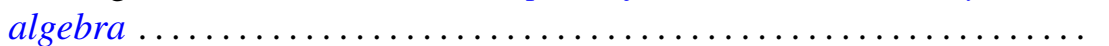

Jacob Feldman and Frederick Paul Greenleaf, Existence of Borel

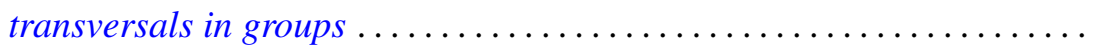

Thomas Muirhead Flett, Mean values of power series 463

Richard Vernon Fuller, Relations among continuous and various

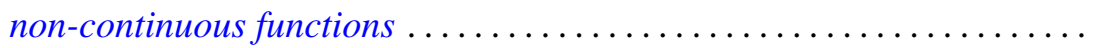

Philip Hartman, Convex sets and the bounded slope condition ............

Marcel Herzog, On finite groups containing a CCT-subgroup with a cyclic Sylow subgroup .

James Secord Howland, On the essential spectrum of Schroedinger

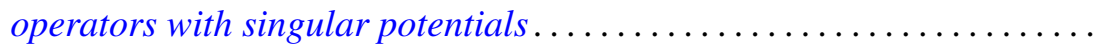

Thomas William Hungerford, On the structure of principal ideal rings .....

Paul Joseph Kelly and Ernst Gabor Straus, Curvature in Hilbert geometries. II mpati Madhusudana Rao, Linear functionals on Orlicz spaces:

Malempati Madhusudana Rao, Linear functionals on Orli
$\quad$ General theory............................
Stanley F. Robinson, Theorems on Brewer sums.......
Ralph Tyrrell Rockafellar, A general correspondence betw

Malempati Madhusudana Rao, Linear functiona
$\quad$ General theory.........................
Stanley F. Robinson, Theorems on Brewer sums
Ralph Tyrrell Rockafellar, A general corresponden

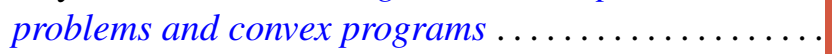

Richard Benjamin Sher, Defining subsets of $E^{3}$ by cubes.

Howard Jacob Weiner, Invariant measures and Cesàro summability... 